\title{
Optimal Electron Beam Sensitivity Determinations Using Live Time Low Dose Imaging of Vitreous Ice and Lens Coupled CCDs
}

\author{
J.F. Mancuso*, Y.M. Heng** and C.A. Ackerley** \\ *Advanced Microscopy Techniques Corp., 3 Electronics Ave, Danvers, MA 01923 \\ ** Division of Pathology, Hospital for Sick Children, Toronto, ON
}

A major restriction in the imaging of biological macromolecules and viruses in vitreous ice in the TEM is specimen damage due to electron exposure. ${ }^{1,2}$ The limits of operation depend on specimen preparation, microscope operation, and detection techniques. Typically high resolution imaging of this kind requires labor intensive conventional photographic methods, which usually permit only one perspective off the field of view being examined. Cryo-imaging usually requires focusing of a remote region of the specimen followed by specimen movement and beam blanking prior to recording the region of interest. ${ }^{2}$ Quantifying low dose behavior may allow the region of interest to be viewed and framed before acquisition and thus avoid transients due to blanking and unblanking of the beam. Comparisons between various techniques and instrumentation are difficult without some kind of standard.

In this study, we have quantified the low dose sensitivity of several CCD cameras in either a side or bottom mount configuration using a fast Fourier transform (FFT) of the live image to detect changes in vitrified ice as an indicator in determining dose limit.

Experimental Procedure

Specimen: Double distilled water was applied to grids with lacey films and the excess water removed by wicking with a filter paper prior to plunge freezing the specimens in liquid ethane. The grids were then cryo-transferred to a Gatan Model 910 cryo-holder, which was maintained at $-150 \mathrm{C}$ in the column.

TEM Operation: In order to determine the electron dose at the specimen, electron density was measured a minimum of 10 times using a holder with Faraday cup in a JEM1230 TEM operating at $100 \mathrm{kV}$. This was repeated a minimum of 10 times over a 2 hour period a minimum of five times in order to determine whether any changes were detectable in current density over time. Microscope conditions were stored in the microscope's operating systems in order to achieve electron doses of 0.5 , 1,5 and 10 electrons $/ \mathrm{A}^{2}$. Vitreous ice was then examined at these doses at a nominal magnification of $100,000 \mathrm{x}$ at the conventional plate film position.

Detector: Three cooled, lens coupled, interline transfer CCDs were used in the following configurations: 1) Side-mounted 1 Mpixel , 2) Side-mounted 5 Mpixel, and 3) Bottom-mounted 6.8 Mpixel. The quantum efficiencies of these configurations are nominally the same. All are well suited for live observations because they have $100 \%$ temporal efficiency (i.e. zero dead time) and can be run in TV format ( $>500$ lines) with viewing rates ranging from 6 to $18 \mathrm{fps}$. Thus the specimen could be monitored for sudden changes. The FFT over a 256x256 pixel center region was computed and displayed for each live frame. 
Conditions FFT images were displayed and monitored from initial specimen irradiation until a diffraction pattern appeared, signifying the onset of specimen deterioration. Experiments were repeated a minimum of 10 times on each camera system. The maximum exposure time was $800 \mathrm{sec}$. Times before changes were observed in the FFT were expressed as a mean and standard error. Specimen dose rates were $0.5,1,5$ and 10 electrons $/ \mathrm{A}^{2} / \mathrm{s}$. A multivariate analysis was done between electron dose conditions and the camera system used to determine significance. In this evaluation live FFT acquisitions were observed on binned images using $2 \times 2$ and $4 \times 4$ binned "super pixels".

\begin{tabular}{|c|c|c|c|c|}
\hline $\begin{array}{l}\text { Camera \& } \\
\text { Conditions }\end{array}$ & $\begin{array}{c}\text { Beam Current } \\
@ \text { @Spec }\end{array}$ & $\begin{array}{c}\text { Dose Rate } \\
\text { @Pixel }\end{array}$ & $\begin{array}{l}\text { Total } \\
\text { Time }\end{array}$ & $\begin{array}{c}\text { Standard } \\
\text { Error }\end{array}$ \\
\hline & $\left(\mathrm{e}^{-} / \mathrm{A}^{2} / \mathrm{s}\right)$ & (e $\mathrm{e}^{-/} / \mathrm{pix} /$ frame $)$ & (s) & $( \pm \%)$ \\
\hline 1 Мрiх & 0.5 & 17 & 543 & 22 \\
\hline side-mount & 1 & 33 & 343 & 19 \\
\hline $2 \times 2$ binning & 5 & 165 & 62 & 50 \\
\hline $18 \mathrm{fps}$ & 10 & 330 & 12 & 69 \\
\hline 5 Мpix & 0.5 & 50 & 598 & 15 \\
\hline side-mount & 1 & 99 & 324 & 30 \\
\hline $4 x 4$ binning & 5 & 496 & 67 & 43 \\
\hline $6 f p s$ & 10 & 991 & 17 & 33 \\
\hline 6.8 Мрix & 0.5 & 1.3 & No FFT & -- \\
\hline bottom-mount & 1 & 2.7 & No FFT & -- \\
\hline $4 x 4$ binning & 5 & 13.4 & 64 & 58 \\
\hline $6 f p s$ & 10 & 26.9 & 19 & 42 \\
\hline
\end{tabular}

Results and Conclusions: The table suggests that there is a dose-rate dependence that is significant at or above $10 \mathrm{e}^{-} / \mathrm{A}^{2} / \mathrm{s}$. The statistical variations for a given dose-rate indicate that the prepared specimens are consistent to about 30\% for the lower dose-rates. There is less consistency at higher dose-rates.

The minimum dose per pixel needed to observe an FFT appears to be between 3 and 13 electrons per super-pixel. This table suggests that there is a reproducible way to measure camera sensitivity with an easily fabricated standard under conditions suitable for cryo-imaging.

Since binning does not affect electrical noise in these systems, then a similar dose per pixel value is needed for normal 1x1 binning. This allows operators to "budget" live viewing and recording exposures by the ratio of the binning factor (squared) even at very low dose rates. Further experiments are planned to accommodate tilt series with the goal of incorporating this approach in optimizing conditions for tomographic reconstructions of biological macromolecules or viruses.

References:

1) C.A. Mannella et al., Proc. Microsc. And Microanal. (1999)416

2) J. Lepault et al., J. Microsc. 129 (1983)89 